\title{
Integrating polymorphism signatures with myocardial perfusion imaging data to improve the prevention of coronary artery disease: Science or science-fiction?
}

\author{
Pierre-Yves Marie, MD, PhD, ${ }^{\mathrm{a}, \mathrm{b}}$ and Sophie Visvikis-Siest, $\mathrm{PhD}^{\mathrm{c}}$ \\ a CHRU-Nancy, Université de Lorraine, Nuclear Medicine \& Nancyclotep Experimental Platform, \\ Nancy, France \\ b Université de Lorraine, INSERM, UMR-1116, Nancy, France \\ c Université de Lorraine, Nancy, IGE-PCV, Nancy, France
}

Received Nov 15, 2020; accepted Nov 16, 2020

doi: $10.1007 / \mathrm{s} 12350-020-02450-4$

See related article, pp. 2909-2916

The last few years have been marked by major advances in the identification of genetic risk factors for coronary artery disease (CAD). Hitherto the predictive benefits of this approach were typically limited to the detection of rare genetic variants such as those involved in familial hyperlipidemia. This advance was ushered in by the identification of disease-specific common singlenucleotide polymorphisms in individual candidate genes as well as variants identified by genome-wide association studies (genome-wide significant variants). Although the predictive value of any individual polymorphism is generally quite low, when multiple polymorphisms are considered together they can yield much higher polygenic risk scores. ${ }^{1}$ A more recent step, which is still in progress, involves polygenic risk scores, which not only analyze genome-wide significant variants and variants from genotyping arrays that exclusively focus on pre-selected loci, but also much larger genome-wide variations, and provide an estimate

Reprint requests: Pierre-Yves Marie, MD, PhD, CHRU-Nancy, Université de Lorraine, Nuclear Medicine \& Nancyclotep Experimental Platform, 54000 Nancy, France; py.marie@chru-nancy.fr J Nucl Cardiol 2022;29:2917-9.

$1071-3581 / \$ 34.00$

Copyright (C) 2021 American Society of Nuclear Cardiology. of the relative contribution of each genetic variant to CAD risk. ${ }^{2}$

Polygenic risk scores for cardiovascular (CV) diseases may query millions of single-nucleotide polymorphisms. Their high predictive value was recently established in several large middle-aged cohorts evaluating primary CAD prevention. ${ }^{3-5}$ However, none of these polygenic scores were found to provide any significant additional value with regard to adjusted risk models based on conventional CV risk factors (age, sex, dyslipidemia, diabetes, obesity....). ${ }^{4,5}$ At the present stage of their development, these polygenic risk scores are therefore not considered to be of any practical interest as an adjunct to conventional programs of primary prevention of $\mathrm{CV}$ diseases and CAD in the general, middle-aged population.

A potential advantage of these polygenic risk scores is that they can be established very early in life, at a time when most conventional CAD-related risk factors are yet to be detected (diabetes, hypertension, dyslipidemia...). This may help modify life trajectories prior to the emergence of cardiovascular risk factors per se and delay the onset of any future $\mathrm{CV}$ events. ${ }^{6}$ Indeed, it is likely that lifestyle and/or pharmacological interventions initiated early in life would be more effective in promoting health and preventing diseases that occur much later in life.

It is important to consider that genome-wide association analyses are to date no longer prohibitive, but streamlined procedures. It is therefore foreseeable that such analyses would, in the not too distant future, be requested by a growing part of the population. This new development would not only be driven by the objective of enhancing $\mathrm{CV}$ risk assessment but more generally, to 
predict the risk of disease, promote health, and prevent diseases over a lifetime.

Moreover, no study has to date reported results on polygenic risk scores as they relate to young individuals. Primary prevention of premature atherosclerotic disease has been unintentionally neglected, as the ability to discriminate risk remains challenging, since age is the most important clinical determinant of a 10 -year risk.

Furthermore, one should not ignore that the transition toward an earlier risk assessment would raise major ethical concerns, with potentially harmful medico-economic impacts, as well as mental health repercussions on healthy individuals with identified risk factors associated with diseases occurring much later in life.

A risk assessment early in life would present an additional advantage, notably the ability to direct programs aiming to increase physical activity, improve nutrition, and prevent smoking to targeted high-risk groups rather than to the general populations, but such an advantage is debatable, and is in any case far from being demonstrated at this stage.

Nevertheless, many patients continue to suffer acute coronary syndromes in the absence of any conventional risk factor. CV risk assessment, therefore, needs to be significantly improved by targeting better and more appropriate preventive measures. In the future, this improvement might involve incorporating more efficient polygenic risk scores with clinical data and specific cardiac imaging techniques able to detect pre-atherogenic stages. In this rapidly evolving environment, the review by Angelidis et al in the current issue of the Journal of Nuclear Cardiology ${ }^{\circledR},{ }^{7}$ is particularly informative. Their analysis leads to the final (and perhaps somewhat optimistic) consideration that "the combination of genetic evidence with the findings of myocardial functional imaging techniques could lead to more efficient screening methods, and may improve decisionmaking with regard to the diagnostic investigation and patients' management.'

More specifically, this article reports a rather detailed and intriguing review of studies evaluating associations between genetic polymorphisms and results from myocardial perfusion (PET) as well as singlephoton emission computed tomography (SPECT) imaging (see Table $1^{7}$ ). These studies provide evidence of distinct relationships between specific polymorphisms, and not only sub-clinical states of decreased myocardial perfusion or coronary flow reserve, but also the potential to improve myocardial perfusion with specific treatments (statins).

This latter point leads us to expect that further improvements in our understanding of genomic variants may help to develop novel risk stratifications for CAD and predict an individual's response to a specific preventive treatment. Myocardial perfusion SPECT and PET imaging may be particularly well adapted to help monitor these responses because of their ability to identify early vascular and endothelial dysfunction before the stage of definite atherosclerotic lesions. Such monitoring would be particularly useful for populations considered to have an increased risk of developing CAD's over the long term. This type of monitoring would be even more compelling if genetic data could help predict the efficiency of preventive treatments leading to a more efficient decision-making in the clinical setting of personalized medicine.

Irrespectively of whether these projections are realistic, or belong to the realm of science-fiction, they nevertheless point out the need for enhanced CV risk estimations, to screen lower risk populations than those currently being investigated by myocardial SPECT and PET perfusion imaging. Further significant progress would be required to adapt the deployment and costeffectiveness of PET to this setting, as well as to further improve diagnostic accuracy and dosimetry for cardiac SPECT imaging.

\section{Acknowledgments}

The authors wish to thank Ms. Petra Neufing for critical review of the manuscript.

\section{Disclosures}

The authors declare that they have no conflict of interest.

\section{References}

1. Lali R, Cui E, Ansarikaleibari A, Pigeyre M, Paré G. Genetics of early-onset coronary artery disease: From discovery to clinical translation. Curr Opin Cardiol 2019;34:706-13.

2. Koyama S, Ito K, Terao C, Akiyama M, Horikoshi M, Momozawa $\mathrm{Y}$, et al. Population-specific and trans-ancestry genome-wide analyses identify distinct and shared genetic risk loci for coronary artery disease. Nat Genet 2020;52:1169-77.

3. Khan SS, Cooper R, Greenland P. Do polygenic risk scores improve patient selection for prevention of coronary artery disease? JAMA 2020;323:614-5.

4. Mosley JD, Gupta DK, Tan J, Yao J, Wells QS, Shaffer CM, et al. Predictive accuracy of a polygenic risk score compared with a clinical risk score for incident coronary heart disease. JAMA 2020;323:627-35.

5. Elliott J, Bodinier B, Bond TA, Chadeau-Hyam M, Evangelou E, Moons KGM, et al. Predictive accuracy of a polygenic risk scoreenhanced prediction model vs a clinical risk score for coronary artery disease. JAMA 2020;323:636-45.

6. Hindy G, Aragam KG, Ng K, Chaffin M, Lotta LA, Baras A, et al. Genome-wide polygenic score, clinical risk factors, and long-term trajectories of coronary artery disease. Arterioscler Thromb Vasc Biol 2020;40:2738-46. 
7. Angelidis G, Valotassiou V, Satra M, Psimadas D, Koutsikos J, Skoularigis $\mathrm{J}$, et al. Investigating the genetic characteristics of CAD: Is there a role for myocardial perfusion imaging techniques? J Nucl Cardiol 2020. https://doi.org/10.1007/s12350-020-02403-x.
Publisher's Note Springer Nature remains neutral with regard to jurisdictional claims in published maps and institutional affiliations. 